\title{
P03-6-12 Poster session
}

\section{Berberine stimulates FGF21 expression via activation of AMP-activated protein kinase in brown and beige adipocytes}

\author{
Yuhei Mitani, Takao Hirai, Michiyo Takagi, Ken-Ichi Nakashima, Makoto Inoue \\ Laboratory of Medicinal Resources, School of Pharmacy, Aichi Gakuin University, Japan
}

Adipose tissues are the major organ that controls systemic energy metabolism and maintain homeostasis. There are two major classes of adipocytes. White adipocytes store and release lipids, while brown adipocytes generate heat by uncoupling oxidative phosphorylation through the action of mitochondrial uncoupling protein 1 . In addition, cold exposure and $\beta 3$ stimulation induce development of brown cell-like "beige" adipocytes in white adipose tissues. Brown adipose tissue and/or beige fat-mediated thermogenesis suppresses high-fat-diet-associated obesity in mice. Therefore, there is great expectation that brown/beige fat activity can be increased to reduce obesity and metabolic disease.

Fibroblast growth factor 21 (FGF21) is an important metabolic regulator of glucose and lipid homeostasis, and is expressed in multiple tissues including liver, pancreas, white and brown adipose tissues, and skeletal muscle. In addition, FGF21 is being pursued as a therapeutic target for diabetes and obesity because of its benefits in regulating glucose and lipid metabolism.

In this study, a total of 88 crude herbal drugs frequently used in Kampo prescriptions (traditional Japanese medicine) were selected and screened for up-regulation of expression of FGF21 in beige adipocytes. As a result, we found that berberine, an isoquinoline alkaloid, significantly increased FGF21 expression in brown and beige adipocytes. Furthermore, we investigated the target molecule of berberine in regulation of the FGF21 expression in beige adipocytes. To address this, total RNA was extracted from in beige adipocytes after exposure to berberine, and analyzed by real time qRT-PCR. We found the expression of FGF21 mRNA was significantly increased by berberine in beige adipocytes. Additionally, the up-regulation of FGF21 mRNA by berberine was completely abolished by an AMP-activated kinase (AMPK) inhibitor, compound $\mathrm{C}$, in beige adipocytes. These findings suggested that berberine stimulated FGF21 expression through activation of AMPK signaling pathway in brown and beige adipocytes. 